\title{
Viewpoints:
}

\section{Current status and development of percutaneous coronary intervention in China ${ }^{*}$}

\author{
Yong HUO \\ (Department of Cardiology and Heart Center, Peking University First \\ Hospital, Beijing 100034, China) \\ E-mail: huoyong@263.net.cn \\ Received June 25, 2010; Revision accepted July 12, 2010 \\ Crosschecked July 13, 2010
}

doi:10.1631/jzus.B1001012

Document code: A CLC number: R54

Dr. Gruntzig et al. (1979) successfully completed the world's first percutaneous coronary intervention (PCI), a percutaneous transluminal coronary angioplasty (PTCA), in 1977. The first reported PTCA in China was in 1984. With the development of PCI techniques, while more and more patients have benefited, there are ongoing challenges as well.

\section{Current development of $\mathrm{PCl}$ in China}

In China, the rate of PCI use has increased by $30 \%$ to $40 \%$ in the last five years. By 2007, 1078 hospitals in 30 provinces completed 144673 interventions (Lu et al., 2008). Due to the specific conditions in China, the development of PCI has its own characteristic challenges. Firstly, there is a tendency for these techniques to be used in concentrated geographical areas. Five geographical areas accounted for 43.2\% of the total PCI's in 2007 (NCCD, 2010). The provinces with the greater PCI usage have been mostly located in the eastern coastal area. As well, standardization of technique has not been emphasized. Because of rapid developments in this area without standards, there have been malpractice cases in some hospitals. Lack of standards increases medical risk, leads to waste of medical resources, and increases costs. Finally, given the large population in China at

\footnotetext{
${ }^{*}$ Project (No. 2006BAI01A02) supported by the National Science and Technology Pillar Program of the 11th five-year Plan, China (C) Zhejiang University and Springer-Verlag Berlin Heidelberg 2010
}

risk for coronary heart disease (CHD), PCI will be required even more in the future.

\section{$2 \mathrm{PCl}$ standardization: contents and goals}

The Ministry of Health (MOH) of China has made considerable efforts towards PCI standardization, particularly through online registration and quality control (QC). The main contents of "standardization" are as follows: certification of institutions and individuals, personnel training before registration, and QC of the diagnostic and therapeutic interventions (official documents on administration website of cardiovascular disease intervention, http:// www.mta.org.cn/). Certification and training guarantee the basic level of competence of institutions and individuals. QC monitors the PCI procedures to hopefully prevent malpractice. Obviously, QC is the most vital means by which to improve PCI care. Currently, training centers have recruited trainees, some of them having finished their courses and having been assessed from September 2009 onward. Under the leadership of government, the national quality control center (QCC) and 31 provincial level branches were established. Different kinds of QC work have been undertaken based on a registry network of QCCs. This article will pool both the online data and the feedback of provincial QCCs in order to provide an initial status report.

\section{$3 \mathrm{PCl}$ data summary of 2009}

According to the document "Notification about Several Case Report Issues in Cardiovascular Interventional Therapy Issued by General Office, $\mathrm{MOH}$ " (official documents on administration website of cardiovascular disease intervention, http://www.mta. org.cn/), all cases of coronary intervention should be reported online within the prescribed time limit. The 
quantity and quality of online case reports will be used to qualify the interventional medical center or the training center. For this first year of direct online reporting, all the cases performed after July 1st in 2009 should be reported in $7 \mathrm{~d}$, and those before July 1st could be reported before Dec. 31st, 2009. In fact, the report deadline was postponed reasonably. By February 2010, a total of 175591 cases were reported online nationwide. Among them, 9\% ( $n=15241)$ were performed in Beijing, 9\% ( $n=15239)$ in Guangdong Province, 7\% ( $n=13035)$ in Shandong Province, 6\% $(n=10831)$ in Shanghai, 6\% $(n=10329)$ in Henan Province, and 6\% $(n=10200)$ in Liaoning Province. About $43 \%$ of cases were reported from these six regions, indicating that cases were highly concentrated in a few of the more developed regions. However, there has been less concentration compared to a few years ago. For example, $15.4 \%$ cases were from Beijing in 2007. In the completed online database containing 169727 cases, excepting the Xinjiang Uygur Autonomous Region, 246756 stents were implanted. The average stent number of each patient is 1.45 (from 0.44 in Gansu Province to 1.95 in Hainan Province). Among them, 95.9\% $(n=236667)$ were drug-eluting stents (from $80.7 \%$ in the Inner Mongolia Autonomous Region to $99.9 \%$ in the Ningxia Hui Autonomous Region). Of these stents, 22.2\% ( $n=$ 54892 ) were foreign made (from $3.6 \%$ in the Inner Mongolia Autonomous Region to $50.3 \%$ in Sichuan Province). The number of mortalities was 559 $(0.32 \%)$. The number of severe complications during or after the procedure was $1503(0.86 \%)$ (database on administration website of cardiovascular disease intervention, http://www.mta.org.cn/).

For many reasons, there might be missing cases of interventional therapy or death. The national QCC sent questionnaires to provincial QCCs to help confirm the numbers of missing cases, dead cases, and cases from non-qualified medical centers. This investigation received active feedback from all the provincial QCCs. Also, the data from 17 army hospitals were added in support of MOH, People's Liberation Army General Logistics Department. Thus, there were 235078 cases in total. Besides $74.6 \%$ of cases $(n=175591)$ reported directly online, $14.0 \%$ cases $(n=32885)$ were missed, and $4.2 \%$ cases ( $n=9707$ ) were performed by non-qualified hospitals. Of all cases, $7.2 \%$ ( $n=16895)$ were reported from 17 army hospitals.

All these 175591 cases were reported by 4389 physicians from 1015 online registered hospitals, although not all of them were qualified. Because the qualification of coronary intervention admission of medical institutions and individuals is still in progress, the number of qualified institutions and individuals is not available yet. Among these 1015 hospitals, more than 140 hospitals did not report any cases, 4 hospitals reported more than 2000 cases (total $n=16704$ ), 22 hospitals reported 1000 to 2000 cases (total $n=28969$ ), 50 hospitals reported 500 to 1000 cases (total $n=34138$ ), and 161 hospitals reported 200 to 500 cases (total $n=50665$ ). Thus, most cases were performed in more than 200 hospitals with case numbers exceeding 200.

\section{Evaluation of $\mathrm{PCl}$ status in 2009}

Based on the registry data of 2009, we found that the PCI case number had increased steadily. The quality of PCI estimated by death rate and incidence of severe complications was very good. In order to improve the quality further, we still need to pay much more attention to the problems resulting from rapid advances in PCI.

Since first calculated systemically in 2006 (NCCD, 2010), the national PCI case number has increased by $25 \%$ to $30 \%$ per year. According to the published data, 144673 cases were performed in 2007, a 28.5\% increase compared to 112580 cases in 2006. About 182312 cases were performed in 2008 (Yuan et al., 2010), a $26.0 \%$ increase compared to 2007. About 240000 cases were performed in 2009, 31.6\% higher than in 2008. Despite increases in case numbers, both the procedure related mortality and complication rate were kept at a reasonable low level. Mortalities reported online were 559 , which was $0.32 \%$ of the total and ranged from 0 to $0.87 \%$ in different provinces. If missing reports of deaths are added, the number of the total mortality cases is $777,0.33 \%$ of all the documented 235078 cases. The incidence of severe complications was $0.85 \%$ ( $0.5 \%$ and $0.35 \%$ for during and post procedure, respectively).

The main problems of online case registry and QC are as follows:

1. Data integrity. Both missing reports and false reports exist. Some cases were missed, and some diagnostic procedures, such as angiography and intravascular ultrasound (IVUS), were reported as therapeutic procedures. Since the process of intervention qualification is not yet completed, each case should be reported no matter if it was performed in a 
qualified hospital or not. The reasons for missing mortality reports are more complicated. Firstly, systematic follow-up of patients who underwent PCI was lacking. Secondly, monitoring and auditing of some provincial QCCs were less intensive. Lastly, the time window of case reporting online should be improved to meet the demand. Data of some reported cases were not intact; for instance, some were lacking history information such as risk factors, and some were lacking procedure details such as the devices used. Thus, hospitals should intensively train the person who will input this essential information. On the other hand, some network limitations are needed to avoid unnecessary data input.

2. Case reporting is not on time. There were only 9 provinces that reported online within $7 \mathrm{~d}$ from the procedure, 14 provinces reported in $30 \mathrm{~d}$, and 6 provinces reported after more than $30 \mathrm{~d}$ (data lacking from Xinjiang Production and Construction Corps and the Xinjiang Uygur Autonomous Region). This makes it difficult for QCCs to find problems, such as consecutive severe complications or even mortalities, and then to resolve them.

3. Indications are not strictly adhered to. Online data showed that unstable angina, non-ST elevation myocardial infarction (NSTEMI), and ST elevation myocardial infarction (STEMI) occupied 54\%, 29\%, and $6 \%$ of the total PCI cases, respectively, $79 \%$ when added together. This ratio is concordant with current guideline recommendations and clinical practice. If considering according to the extent of the lesion stenosis, only $65 \%$ of them ranged from $75 \%$ to $99 \%$, and $12 \%$ of them were less than $50 \%$. Although intervention for a lesion less than $50 \%$ is acceptable for some reasons, the reported rate is higher than expected. Stricter QC of these kinds of cases is required.

4. Primary PCI for STEMI requires further development. Only 50888 (29\%) of STEMI patients underwent PCI in 2009. This number was quite low because hundreds of thousands of acute myocardial infarctions (AMIs) occur in China each year. In addition, there were only 15221 primary PCI cases among them, less than $1 \%$ of the total AMI patients (NCCD, 2010). Also, we cannot tell how many cases met the "door to balloon" time within the 90 min standard. Because primary PCI can improve both the acute and long-term prognoses as well as the quality of life in patients, a series of strategies for diagnosis, patient transfers, and technique details should be upgraded to benefit more patients.
As a novel organizing model, online registration and QC of PCI cases should be continuously improved. It is recommended to strengthen the related issues at different levels: (1) Support from health administrative departments is the key. Authentication and publicity of institutions and individuals qualification should be completed as soon as possible by health administrative departments at all levels. Most especially, the qualification of second order hospitals should be resolved. (2) QCCs at different levels, especially in provincial QCCs, should take solid measures to manage and register, as well as supervise, inspect, and audit the PCI cases more seriously. Online case reports should be accurate and timely. QCCs need to discover and resolve problems to ensure the quality of PCI. (3) Improvement of the web platform is essential. The platform should allow for easy and quick data input. Database managements, such as data off-line input and searching tools, are necessary. Special techniques should be used to guarantee data accuracy, for example, setting necessary limitations to forbid illegal data input.

In the future, with the support of health administrative departments, specialist committees, and hospitals, every QCC should hold a "solidarity and harmonizing" spirit to "standardize" PCI and to scientifically advance the development of PCI techniques and QC.

\section{Acknowledgements}

Special thanks to Dr. Zhao-ping LIU and Dr. Yan ZHANG (Department of Cardiology and Heart Center, Peking University First Hospital, Beijing, China) for the data analyzing of the manuscript.

\section{References}

Gruntzig, A.R., Senning, A., Siegenthaler, W.E., 1979. Nonoperative dilatation of coronary artery stenosis: percutaneous transluminal coronary angioplasty. The New England Journal of Medicine, 301(2):61-68.

Lu, S.Z., Chen, Y.D., Song, X.T., 2008. Memorandum of Coronary interventional salon of China. Chinese Journal of Cardiology, 36(6):563-564 (in Chinese).

NCCD (National Center for Cardiovascular Diseases, China), 2010. Report on Cardiovascular Disease in China (2008-2009). Encyclopedia of China Publishing House (in Chinese).

Yuan, F., Song, X.T., Lu, S.Z., 2010. Percutaneous coronary intervention in mainland China in 2008: registry results. Chinese Journal of Cardiology, 38(7):629-632 (in Chinese). 